\title{
QUALIDADE DE VIDA EM PACIENTES HEMODIALÍTICOS: AVALIAÇÃO ATRAVÉS DO QUESTIONÁRIO KDQOL-SFTM
}

\author{
QUALITY OF LIFE IN HEMODIALYTIC PATIENTS: EVALUATION THROUGH \\ THE KDQOL-SF ${ }^{\text {TM }}$ QUESTIONNAIRE
}

\author{
Camila Zanesco', Elisangela Giachini', Cesar Augusto França Abrahão², Débora Tavares de \\ Resende e Silva'
}

\begin{abstract}
Unive Abstract

Introduction: Chronic Renal Disease (CKD) is understood as a clinical syndrome characterized by the gradual and progressive reduction of excretory, endocrine and metabolic renal functions. Objective: To evaluate the quality of life in patients with CKD on hemodialysis. Methods: Exploratory, longitudinal study with analytical quantitative analysis. Data collected in the period from February to June 2015, at the Reference Nephrology Unit of the western region of Santa Catarina. The instrument used was the questionnaire: KDQOL-SF ${ }^{\mathrm{TM}} 1.3$ (Kidney Disease Quality of Life - Short Form 1.3). Results: 42 patients participated, $52.38 \%$ were males and $57 \%$ of the total sample presented in adulthood. The "emotional performance" dimension obtained a higher mean score (92.06) followed by the "encouragement of dialysis staff" dimension (91.67); the "sexual function" dimension had a lower average score (23.81) followed by "general health" (39.40).Conclusion: It was observed that at the beginning of the Treatment the patients present physical and later emotional changes. It is up to health professionals and their families to deal with patients with CKD in hemodialysis treatment, to understand the same as singular, to recognize their real potentialities and fragilities, in order to trace care and plans directed to the real situation of the patient.
\end{abstract}

Key words: Coresidence concept, elderly people, cognitive analysis, semantic networks.

\section{Resumo}

Introdução: Doença Renal Crônica (DRC) é compreendida como síndrome clínica caracterizada pela redução, gradual $e$ progressiva das funções renais excretoras, endócrinas e metabólicas. Objetivo:Avaliar a qualidade de vida em pacientes com DRC em hemodiálise. Métodos: Estudo de caráter exploratório, longitudinal, com análise quantitativa analítica. Dados coletados no período defevereiro à junhode 2015, na Unidade Nefrológica de referência da região oeste de Santa Catarina. O instrumento utilizado foi $o$ questionário: KDQOL-SF ${ }^{\mathrm{TM}} 1.3$ (Kidney Disease Quality of Life - Short Form 1.3). Resultados: Participaram 42 pacientes, destes 52,38\% eram do sexo masculino e $57 \%$ da amostra total apresentou-se em idade adulta. A dimensão "desempenho emocionais" obteve escore médio mais alto(92,06) seguido da dimensão "encorajamento do pessoal da diálise" $(91,67) ;$ já a dimensão "função sexual" obteve escore médio mais baixo $(23,81)$ seguido de "saúde em geral" $(39,40)$. Conclusão:Observou-se que no inicio do tratamento os pacientes apresentam alterações físicas e posteriormente emocional. Cabe aos profissionais de saúde e os familiares que lidam com pacientescom DRC em tratamento hemodialítico, entender o mesmo como singular, reconhecer suas reais potencialidades $e$ fragilidades, para desta forma traçar cuidados e planos direcionados diante da real situação do doente.

Palavras chave: Corresidência, idoso, análise cognitiva, redes semânticas 


\section{Introdução}

O aumento da expectativa de vida populacional advindos da evolução tecnológica e científica, propicia o desenvolvimento de doenças crônicas não transmissíveis (DCNT) gerando causas de mortes prematuras no mundo que afeta a qualidade de vida (QV). Esse fato é favorecido pelas limitações nas atividades laborais, recreativas, econômicas ao grupo familiar, comunidade e a sociedade em geral, agravando as iniquidades e aumentando a pobreza'.

DCNT são impactantes no processo de morbidade e mortalidade da população em geral $^{2}$. A Doença Renal Crônica (DRC) faz parte deste grupo de DCNT e é compreendida como uma síndrome clínica caracterizada pela redução significativa, lenta, gradual e progressiva das funções renais excretoras, endócrinas e metabólicas $^{3}$. A etiologia da DRC permanece desconhecida, é fortemente associada a doenças sistêmicas como a Hipertensão Arterial, Diabetes Melittus e glomérulopatias ${ }^{4}$.

A DRC é caracterizada por aparecimento de lesões renais, podendo ou não ser associada a decréscimo na Taxa de Filtração Glomerular (TFG) para valores inferiores a $60 \mathrm{~mL} / \mathrm{min} / 1,73$ $\mathrm{m}^{2}$ durante períodos de três ou mais meses ${ }^{5}$. Quando a TFG apresenta-se inferior a 15 $\mathrm{ml} / \mathrm{min} / 1,73 \mathrm{~m}^{2}$, o paciente está na fase terminal ou dialítica, restando à Terapia Renal Substitutiva (TRS), disponível nas seguintes modalidades: diálise peritoneal ambulatorial continua (DPAC), diálise peritoneal intermitente (DPI), diálise peritoneal automatizada (DPA), hemodiálise (HD) e o transplante renal, sendo a HD a mais usada $^{6,7,8}$.

Essas opções associadas a mudanças nos hábitos alimentares e de vida, acarretam na minimização dos sintomas e na preservação da vida dos pacientes, entretanto nenhum dos tratamentos mencionados acarreta na cura ${ }^{9}$. No estágio em que a atividade renal normal é reduzida em mais de $90 \%$, a ausência de tais intervenções podem levar o paciente a óbito em até 72 horas $^{10}$.

A escolha da TRS é realizada pelo nefrologista, sendo que o tratamento mais utilizado é a hemodiálise $(H D)^{11}$. No primeiro trimestre de 2015 encontravam-se na lista de espera para transplante cerca de 18.818 doentes, porém este dado só representa cerca de $17 \%$ dos aproximadamente 110.000 pacientes em TRS no Brasil $^{12}$.

Desde a revelação do diagnóstico e da rotina de tratamento, ocorrem alterações psíquicas e emocionais, advindas do isolamento social, alterações funcionais do paciente o que traz limitações as atividades de vida diária e abandono da atividade laboral ${ }^{4}$.

O conjunto de inúmeras mudanças adaptativas impostas pela DRC aos pacientes impactam em alterações na QV dos mesmos ${ }^{2}$; sendo que QV é definida pela Organização Mundial De Saúde como: "a percepção do indivíduo de sua posição na vida no contexto da cultura e sistema de valores nos quais ele vive e em relação aos seus objetivos, expectativas, padrões e preocupações". Sendo assim, é importante considerar a necessidade de avaliar a QV, em pacientes portadores de DRC submetidos ao tratamento hemodialítico.

A presença da ansiedade acompanhada de opressão dolorosa perturba a consciência do ser, assim, agonia, ansiedade, apreensão e aperto tornam-se características emocionais marcantes na forma de expressão do desconforto presente no paciente DRC. Dessa forma, notar tais alterações emocionais e físicas por critérios avaliativos direcionam equipes multiprofissionais a uma melhor abordagem e direcionamento no acompanhamento do tratamento e compreensão frente às restrições individuais e em sua rotina.

O Ministério da Saúde do Brasil ${ }^{13}$, ressalta que a DRC acomete $10 \%$ da população, estimando que atinja um a cada cinco homens e uma a cada quatro mulheres com idades entre 65 e $74 \operatorname{anos}^{14}$. Nesse sentido, sabendo que a QV contribui, influência e direciona equipes multiprofissionais da área de saúde no acompanhamento e tratativa do paciente com DRC, foi proposto avaliar pacientes com DRC em tratamento por HD.

\section{Metodologia}

A pesquisa realizada foi de caráter observacional, exploratório, longitudinal, com análise quantitativa analítica. A presente pesquisa ocorreu em um serviço de hemodiálise do Oeste catarinense, o qual é referência para mais de 30 municípios, aos quais são ofertadas as seguintes modalidades de tratamento: HD, transplante renal e atendimento para pós transplante. O período de realização da pesquisa foi de fevereiro a junho do ano de 2015.

As informações necessárias para a pesquisa, referente aos pacientes, foram coletadas através de entrevista, guiada pelo instrumento: o questionário KDQOL-SF ${ }^{\mathrm{TM}} 1.3$ (Kidney Disease 
Quality of Life - Short Form 1.3). Foram abordados os pacientes dos três turnos diários que acontecem na clínica. Todos responderam o questionário. Este questionário abrange 19 dimensões, possui 43 perguntas específicas da DRC e 36 perguntas do foro genérico, além de uma pergunta de identificação geral de saúde ${ }^{15}$.

Os pacientes deveriam estar realizando HDno mínimo há 03 meses e no máximo há 36 meses,e assinar o Termo de Consentimento Livre e Esclarecido (TCLE) para serem incluídos na pesquisa. Foram excluídos pacientes que foram submetidos a transplante renal e/ou vieram a falecer no período da pesquisa.

Para a análise dos dados foi utilizado instrumento/planilha disponível online e gratuita para análise do KDQOL-SF ${ }^{\mathrm{TM}} 1.3$.

O trabalho foi aprovado pelo Comitê de Ética em Pesquisa (CEP) da Universidade Federal da Fronteira Sul (UFFS), sob o protocolo CAAE:33713514.7.0000.5564. Os pacientes foram informados sobre a forma do estudo e aceitaram participar de cunho voluntario - conforme determina a Resolução n. 196/96 do Conselho Nacional de Saúde, que discorre acerca da pesquisa envolvendo seres humanos - dos protocolos de coleta de dados (questionários) do estudo. Foi realizado contato de forma formal com os diretores responsáveis pela clínica selecionada para o desenvolvimento da pesquisa no âmbito de obter apoio e autorização necessários à realização da aplicação do questionário.

\section{Resultados}

A amostra final foi composta por 42 pacientes, sendo que destes 20 pacientes (47.62\%) eram do sexo feminino e 22 pacientes (52.38\%) eram do sexo feminino. Quanto a idade observamos que 24 pacientes (57\%) eram adultos e tinham entre19 a 64 anos, os demais eram idosos e destes, 15 pacientes (36\%) tinham entre65 a 80 anos e3 pacientes (7\%) tinham mais que 80 anos. Todos os participantes eram de etnia caucasiana, nenhum possuía hepatite B ou C e todos eram HIV negativos.

A duração das sessões variou entre três (03) horas a quatro (04), sendo que todos os participantes da pesquisa realizam três (03) sessões semanais de HD. Considerando o tempo de tratamento por HD obtivemos os seguintes resultados: entre 08 e 12 meses foram 13 pacientes (30,95\%), 03 e 07 meses foram 11 pacientes (26,19\%), 25 e 36 meses foram 10 pacientes $(23,81 \%)$, e 13 e 24 mesesforam8 pacientes $(19,05 \%)$.

Os dados obtidos em relação a Qualidade de Vida (QV), avaliada através da utilização do instrumento, o questionário KDQOL-SF ${ }^{\mathrm{TM}} 1.3$ são apresentados na Tabela 1.

Tabela 1- Avaliação da qualidade de vida através do questionárioKDQOL-SF ${ }^{\mathrm{TM}} 1.3$ :

\begin{tabular}{lll}
\hline Dimensões & Média & $\mathbf{N}^{\circ}$ \\
KDQOL-SFTM1.3 & & 42 \\
(n de questões das dimensões) & 75,20 & 42 \\
Sintomas/problemas (12) & 72,92 & 42 \\
Efeitos da doença renal na vida diária (8) & 42,41 & 42 \\
Peso da doença renal (4) & 82,14 & 42 \\
Atividade profissional (2) & 79,68 & 42 \\
Função cognitiva (3) & 61,27 & 21 \\
Qualidade da interação social (3) & 23,81 & 42 \\
Função sexual (2) & 69,40 & 42 \\
Sono (4) & 56,75 & 42 \\
Apoio social (2) & 91,67 & 42 \\
Encorajamento do pessoal da diálise (2) & 82,14 & 42 \\
Satisfação do doente (1) & 54,52 & 42 \\
Aspectos físico (10) & 87,50 & 42 \\
Desempenho físico (4) & 57,80 & 42 \\
Dor (2) & 39,40 & 42 \\
Saúde em geral (5) & 65,62 & 42 \\
Aspectos emocionais (5) & 42 \\
Desempenho emocionais (3) & 92,06 & 42 \\
Aspectos sociais (2) & 56,55 & \\
Vitalidade (4) & 57,38 & \\
\hline Fonte: autores, 2016. & & \\
\hline
\end{tabular}

Fonte: autores, 2016. 


\section{Discussão}

Avaliar a QV de pacientes portadores de DRC em HD se mostra de suma importância para poder auxiliar na condução de melhores abordagens clínicas, verificar a necessidade de acompanhamento psicológico para avaliar alterações emocionais, assim como fisioterapêutico para aliviar as questões físicas. Sendo assim, conhecer quem são os pacientes envolvidos se torna imprescindível para condutas clínicas. Neste estudo constatou-se que foi encontrada discreta predominância do sexo masculino $52,38 \%$ nos pacientes abordados, o fato condiz com pesquisas já publicadas que encontraram uma prevalência de $68 \%$ de indivíduos do sexo masculino ${ }^{15}$.

Nossos dados mostraram prevalência de pacientes com DRC em idade adulta, ou seja, pacientes com mais de 19 anos, resultado que condiz com o cenário nacional exposto em publicações oficiais ${ }^{16}$ da Sociedade Brasileira de Nefrologia $^{12}$, assim como é verificado em resultados de diversos estudos ${ }^{5,17,20,21}$.

No que tange a duração das sessões de HD, a mesma é adequada de acordo com as necessidades de cada um dos pacientes, visto que esta adequação já esta preconizada nos serviços. Em relação ao tempo de tratamento e a delimitação do público estudado em nossa pesquisa, foi-se abordado pacientes que estavam em tratamento por HD no mínimo há 03 meses e no máximo por 36 meses. Em outro estudo de caráter transversal observou-se a participação de 156 pacientes e a média foi de 38 meses de tratamento $^{17}$.

O estudo de Cavalcanti, $2013^{5}$ demonstra que as dimensões com menor escore de QV foram: situação ocupacional, peso da doença renal, saúde geral, satisfação do paciente e função física, resultado que vai de encontro com os obtidos no presente estudo, pois observamos que o desempenho da saúde em geral obteve um dos nossos menores escores.

Nas questões relacionadas a "aspectos físicos" avaliou-se as limitações e a intensidade para os tipos e quantidade de trabalho, ou outras atividades executadas, além das queixa/respostas mais frequentes, e observou-se que as respostas estavam relacionadas a fraqueza física, fadiga, mal-estar e desconforto geral com o tratamento, o mesmo observado em outros estudos 5 .

Os distúrbios do sono não são fatores de extremo incomodo para os pacientes com DRC em TRS por HD na região oeste catarinense o que difere do apontado em estudos publicados que apontam os distúrbios relacionados ao sono como interferência negativa para a $\mathrm{QV}^{21}$.

No domínio da saúde geral os níveis obtidos foram diminuídos, resultado encontrado em estudos já publicados ${ }^{21}$. O paciente de DRC convive diariamente com a negação e principalmente sofre com as consequências impostas pela doença e sua evolução, além de ter de se submeter a um tratamento doloroso, com inúmeras limitações e alterações que repercutem na sua própria $\mathrm{QV}^{22}$; Nesse sentido um estudo previamente realizado que utilizou o KDQOL-SF ${ }^{\mathrm{TM}} 1.3$, encontrou apenas um único domínio abaixo de 50, que foi o peso da doença renal ${ }^{5}$.

Na dimensão relacionada a função sexual, observou-se que nem todos os entrevistados se sentiram confiantes e/ou confortáveis para responder as questões, sendo que os participantes que responderam contribuíram para a obtenção de um resultado negativo, apontados em outros estudos já publicados.

Avaliar a QV de vida e identificar os pontos possíveis de serem alterados e/ou melhorados na vida diária de um paciente em HD, auxilia na maior efetividade do tratamento, bem como acarreta na diminuição de gastos com hospitalizações ${ }^{5}$. O DRC convive com uma doença incurável com tratamento desgastante e rotineiro para aliviar/minimizar os sintomas. Dessa forma os mínimos detalhes se fazem imprescindíveis ${ }^{22}$.

\section{Conclusão}

A pesquisa buscou avaliar a qualidade de vida (QV) através do instrumento, o questionário KDQOL-SF ${ }^{\mathrm{TM}} 1.3$ em pacientes com DRC e que realizam HD em uma Unidade Nefrológica no oeste de Santa Catarina. Destacamos a receptividade no serviço e, por parte da equipe de enfermagem e profissionais médicos, bem como da aceitação por parte dos pacientes de participação, questões simples que facilitam e resultam positivamente no contexto geral da pesquisa.

Sugere-se novos estudos sobre a QV de pacientes DRC em tratamento por HD através de outros questionários disponíveis, como o SF-36 e questionário sócio econômico demográfico, além de sugerir um estudo com maior abrangência, afim de relacionar as dimensões do KDQOL$\mathrm{SF}^{\mathrm{TM}} 1.3 \mathrm{com}$ itens destes outros questionários, por exemplo, abranger sexo, ocupações e outras 
questões apontadas em alguns estudos como, fatores esses que influenciam na QV.

Com nossos dados, conclui-se que no inicio do tratamento os pacientes apresentam alterações físicas e posteriormente,o aspecto mais acometido é o emocional. Cabe aos profissionais de saúde e os familiares que lidam com pacientescom DRC em tratamento por HD, entender o mesmo como singular, reconhecer suas reais potencialidades e fragilidades, para desta forma traçar cuidados e planos direcionados diante da real situação do doente.

\section{Referências}

1. BRASIL. Ministério da Saúde. Secretaria de Vigilância em Saúde. Departamento de Análise de Situação de Saúde. Plano de ações estratégicas para o enfrentamento das doenças crônicas não transmissíveis (DCNT) no Brasil 2011-2022 / Ministério da Saúde. Secretaria de Vigilância em Saúde. Departamento de Análise de Situação de Saúde. - Brasília: Ministério da Saúde, 2011.

2. TERRA FDS. Avaliação da qualidade de vida do paciente renal crônico submetido à hemodiálise e sua adesão ao tratamento farmacológico de uso diário. Dissertação (Mestrado) - Curso de Mestre em Saúde, Coordenação de Pesquisa e Pós-graduação, Universidade José do Rosário Vellano, Alfenas, 2007. 175p.

3. BARBOSA ACSCS, SALOMON ALR. Resposta inflamatória de pacientes com doença renal crônica em fase pré-dialítica e sua relação com a ingestão protéica. Com. Ciências Saúde. 2013; 22(4):111-125.

4. HIGA K, KOST MT, SOARES DM, MORAIS MCD, POLINS BRG. Qualidade de vida de pacientes pacientesdensuficiência renal crônica em tratamento de hemodiálise. Acta Paul Enferm2008;21(N'mero Especial):203-6.

5. CAVALCANTE ZC. Fatores associados à qualidade de vida de adultos em hemodiálise em uma cidade do nordeste do Brasil. J BrasNefrol 2013;35(2):79-86.

6. BASTOS MG, BREGMAN R, KIRSZTAJN GM. Doença renal crônica: frequente e grave, mas também prevenível e tratável. RevAssocMedBras 2010; 56(2): 248-53.

$7 . \quad$ STASIAK CES,Bazan KS, KussRS,Schuinski AFM, Baroni G. Prevalência de ansiedade e depressão e suas comorbidades em pacientes com doença renal crônica em hemodiálise e diálise peritoneal. J. Bras. Nefrol. vol.36 no.3
São Paulo Jul/Set. 2014.

8. PATAT, $\mathrm{Cl}$ et al. Análise da qualidade de vida de usuários em hemodiálise. Enfermaria Global, Onlinne, v. 2, n. 27, p.66-76, jul. 2012.

9. SOARES CB, OCHIRO EY, SANNOMIYA NT. Relação da temperatura da solução de diálise e a hipotensão arterial sintomática observada durante sessões de hemodiálise em pacientes com insuficiência renal crônica. Ver EscEnferm USP 2001; 35(4): 346-53.

10. COSTA PBD, VASCONCELOS KFDS, TASSITANO RM. Qualidade de vida: pacientes com insuficiência renal crônica no município de Caruaru, PE. Fisioter. Mov., Curitiba, v. 23, n. 3, p. 461-471, jul./set. 2010.

11. COSTA FG, COUTINHO MDPDL, SANTANA IOD. Insuficiência renal crônica: representações sociais de pacientes com e sem depressão. Psico-USF, Bragança Paulista, v. 19, n. 3, p. 387-398, set./dez. 2014.

12. Registro Brasileiro de Transplantes. Associação Brasileira de Transplantes de Órgãos. Dados da Sede da ABTO. São Paulo - SP. 2015.

13. Ministério da Saúde do Brasil. (2015). Doença renal crônica atinge $10 \%$ da população mundial. Brasília: DF: Portal Brasil.

14. SILVA G, et al. Percepção de portadores de doença renal crônica com relação ao tratamento hemodialítico. INTESA (Pombal - PB Brasil) v. 9, n. 1, p. 23-30 Jan. - jun., 2015.

15. LOPES JM, FUKUSHIMA LRM, INOUYE K, PAVARINI SCI, ORLANDI FDS. Qualidade de vida relacionada à saúde de pacientes renais crônicos em diálise. Acta Paul Enferm. 2014; 27(3):230-6.

16. SESSO, Ricardo Cintra, et al. Relatório do Censo Brasileiro de Diálise Crônica 2012. J. Bras. Nefrol, São Paulo, v. 36, n. 1, 2014.

17. PINTO, Ana Paula et al. Impact of hemodialysis session on handgrip strength. Jornal Brasileiro de Nefrologia, [s.l.], v. 37, n. 4, p.451-457, jul. 2015.

18. FERREIRA PL, ANES EJ. Medição da qualidade de vida de insuficientes renais crónicos: criação da versão portuguesa do KDQOL-SF. Revista Portuguesa De Saúde Pública JANEIRO/JUNHO 2010. Vol28 N. 1: 31-40.

19. ABREU IS, SANTOS CBD. Qualidade de vida relacionada à saúde de pacientes em hemodiálise. Rev. enferm. UERJ, Rio de Janeiro, $2013 \mathrm{jan} / \mathrm{mar} ; 21(1): 95-100$.

20. FERREIRA RC, FILHO CRDS. A qualidade de vida dos pacientes renais crônicos em hemodiálise na região de Marília, São Paulo.JBrasNefrol 2011;33(2):129-135[artigo 
original].

21. GUIMARÃES CKD, Alves DAG , Guimarães LHDCT. Avaliação da qualidade e quantidade do sono em pacientes renais crônicos submetidos à hemodiálise. RevNeurocienc2011;19(4):609-613 [artigo original].

22. FRAZÃO CMFDQ, RAMOS VP, LIRA ALBDC. Qualidade de vida de pacientes submetidos a hemodiálise. Rev. enferm. UERJ, Rio de Janeiro, 2011 out/dez; 19(4):577-82 [artigo de pesquisa].

Endereço para Correspondência

Universidade federal da Fronteira do Sul

e-mail: camila_zanesco@hotmail.com

Recebido em 13/10/2016

Aprovado em 09/03/2017

Publicado em 11/05/2017 